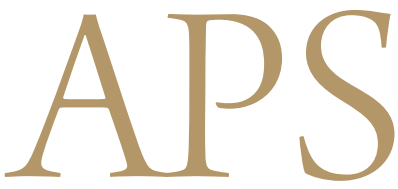

Archives of Plastic Surgery

\title{
Potential Use of Transferred Lymph Nodes as Metastasis Detectors after Tumor Excision
}

\author{
Fabio Nicoli ${ }^{1,2,3}$, Pedro Ciudad ${ }^{1}$, Seong Yoon $\operatorname{Lim}^{1}$, Davide Lazzeri ${ }^{3}$, Christopher D'Ambrosia ${ }^{1}$, \\ Kidakorn Kiranantawat ${ }^{1,4}$, Ram M Chilgar ${ }^{1}$, Stamatis Sapountzis ${ }^{1}$, Bulent Sacak ${ }^{1}$, \\ Hung-Chi Chen ${ }^{1}$ \\ ${ }^{1}$ Department of Plastic and Reconstructive Surgery, China Medical University Hospital, Taichung, Taiwan; ${ }^{2}$ Department of Plastic and \\ Reconstructive Surgery, University of Rome Tor Vergata, Roma; ${ }^{3}$ Plastic, Reconstructive, and Aesthetic Surgery, Villa Salaria Clinic, Rome, \\ Italy; ${ }^{4}$ Department of Plastic and Maxillofacial Surgery, Faculty of Medicine, Ramathibodi Hospital, Mahidol University, Bangkok, Thailand
}

Due to the fact that it reliably results in positive outcomes, lymph node flap transfer is becoming an increasingly popular surgical procedure for the prevention and treatment of lymphedema. This technique has been shown to stimulate lymphoangiogenesis and restore lymphatic function, as well as decreasing infection rates, minimizing pain, and preventing the recurrence of lymphedema. In this article, we investigate possible additional benefits of lymph node flap transfer, primarily the possibility that sentinel lymph nodes may be used to detect micrometastasis or in-transit metastasis and may function as an additional lymphatic station after the excision of advanced skin cancer.

Keywords Lymph node / Free tissue flaps / Melanoma / Carcinogenesis / Lymphedema
Correspondence: Hung-Chi Chen Department of Plastic and Reconstructive Surgery, China Medical University Hospital, 2 Yuh-Der Road, Taichung, 40447, R.0.C, Taiwan Tel: +886-4-2205-2121 (1538) Fax: +886-4-2202-0038 E-mail: D19722@mail.cmuh.org.tw

No potential conflict of interest relevant to this article was reported.

Received: 4 Jan 2015 • Revised: 6 Apr 2015 • Accepted: 7 Apr 2015

pISSN: 2234-6163 • elSSN: 2234-6171 • http://dx.doi.org/10.5999/aps.2015.42.4.478• Arch Plast Surg 2015;42:478-483

\section{INTRODUCTION}

Due to the fact that it reliably results in positive outcomes, lymph node flap transfer (LNFT) is becoming an increasingly popular surgical procedure for the prevention and treatment of lymphedema. This technique has been shown to stimulate lymphoangiogenesis and restore lymphatic function, as well as decreasing infection rates, minimizing pain, and preventing the recurrence of lymphedema $[1,2]$. More specifically, recent studies have demonstrated that lymphatic drainage may be improved and/or restored by transferring vascularized lymph nodes from the supraclavicular, groin, axillary, submental, or abdominal areas into the distal parts of lymphedematous limbs [3-5].

Human lymph nodes have the capacity to enhance the regen- eration of the lymphatic network and stimulate lymphoangiogenesis by releasing the VEGF-C growth factor [6].

In order to prevent upper limb lymphedema, LNFT may also be used as an adjunctive therapy in cases of breast reconstruction [4]. A number of lymphatic restoration techniques may also be utilized as a prophylactic measure after lymph node dissection $[7,8]$.

While the clinical picture of lymphedema is often complicated by recurrent infections of the affected limb, lymph node transfer has been shown to enhance the immunological status of lymphedema patients and reduce infection rates [1,6]. Moreover, in patients with upper limb lymphedema, lymph node transplantation has the advantage of reducing neuropathic pain in the arm [1]. 
In this study, we investigated potential additional benefits of LNFT, primarily the possibility that sentinel lymph nodes may be used to detect micro-metastasis or in-transit metastasis and function as an additional lymphatic station after the excision of advanced skin cancer [9].

\section{IDEA}

\section{Patient population}

We retrospectively analyzed a series of six patients who underwent surgical resection for advanced malignant tumors between October 2013 and June 2014. All patients underwent LNFT either to prevent or to treat early-stage lymphedema. The average age of the patients was 49.6 years, with a range of 33-60 years. All patients underwent dissection of the axillary or groin lymph nodes for palpable nodules, depending on the location of the tumor. Three patients underwent excision of skin cancers (melanoma or squamous cell carcinoma) and primary reconstruction using a groin flap and LNFT. Three patients underwent excision of a melanoma and reconstruction with a lateral arm osteocutaneous flap, a medial sural flap, and a gluteal fasciocutaneous flap. Between one and three months postoperatively, these patients developed subclinical lymphedema and were admitted to the hospital for a secondary surgical treatment with LNFT. Six months following the LNFT procedure, all patients received a positron emission tomography/computed tomography (PET/CT) scan and lymphoscintigraphy in order to determine whether overall metastatic disease and lymphedema of the limb were present. At the same time, we examined the transferred lymph nodes to determine whether metastatic fragments were found in the flap. Fluorescence lymphography using indocyanine green was performed to investigate the lymph flow. Contrast fluid was injected intracutaneously into the distal limb and an infrared camera system was used to obtain images of the superficial lymphatic flow within a few minutes after the injection. After a mean of 7.5 months of follow-up, one patient showed lung metastasis. No metastasis was present in the transferred lymph node flaps.
All limbs had symmetrical circumferences, with no significant swelling present. Clinical data about all patients are presented in Table 1.

\section{Case 1}

A 33-year-old male was admitted for the treatment of an acral melanoma of the right thumb after a positive excision biopsy (Fig. 1). Radical excision with preservation of the metacarpophalangeal joint was performed along with dissection of the palpable axillary lymph nodes. A lateral arm osteocutaneous flap was raised, and the posterior radial collateral artery and its concomitant veins were anastomosed to the dorsalis pollicis artery, its concomitant vein, and a tributary of the cephalic vein. After two months, the patient returned to the outpatient clinic with swelling of the right arm. Lymphoscintigraphy confirmed subclinical lymphedema, and a LNFT was planned. A groin lymph node flap based on the superficial circumflex artery and vein was transferred to the volar site of the right wrist and anastomosed with the superficial palmaris branch of the radial artery, its concomitant vein, and a superficial branch of the cephalic vein. At six months after the operation, PET/CT showed survival of the LNFT, and no metastatic disease was evident in the

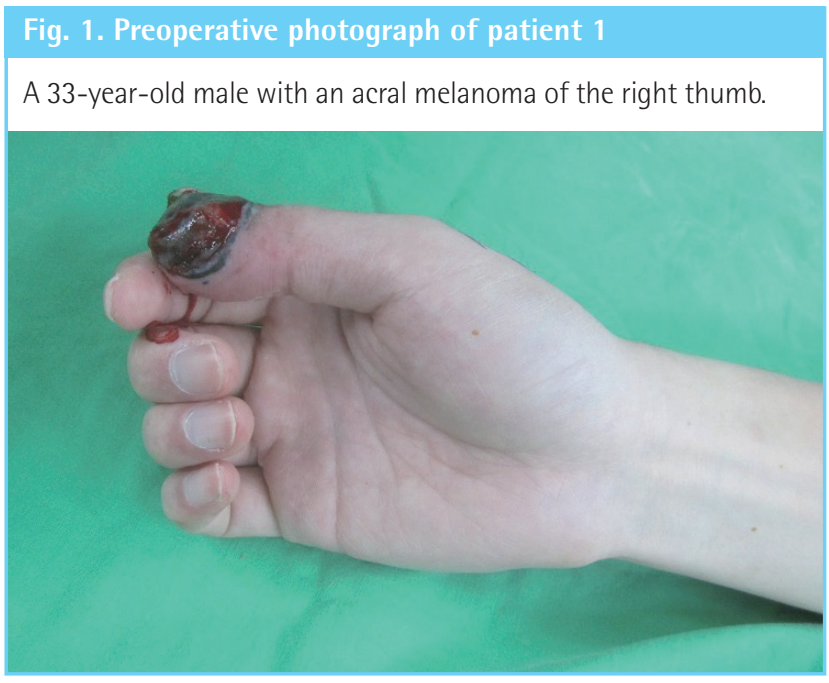

\section{Table 1. Clinical data pertaining to the patients in this study}

\begin{tabular}{lcccc}
\hline Patient & Sex/Age $(\mathrm{yr})$ & Tumor site & Flap coverage+LNFT & Type \& stage tumor \\
\hline 1 & Male/33 & Thumb & Lateral arm flap+GLNF & Melanoma III C \\
2 & Female/53 & Foot & Groin flap+GLNF & Melanoma III C \\
3 & Male/60 & Foot & Medial sural flap+SLNF & Melanoma III B \\
4 & Male/52 & Foot & Groin flap+LN & Poorly differentiated SCC \\
5 & Male/46 & Hand & Groin flap+LN & Melanoma III B \\
6 & Male/54 & Foot & Gluteal flap+SLNF & Melanoma III C \\
\hline
\end{tabular}

LNFT, Iymph node flap transfer; GLNF, groin lymph node flap; SLNF, supraclavicular lymph node flap; LN, lymph nodes; SCC, squamous cell carcinoma. 


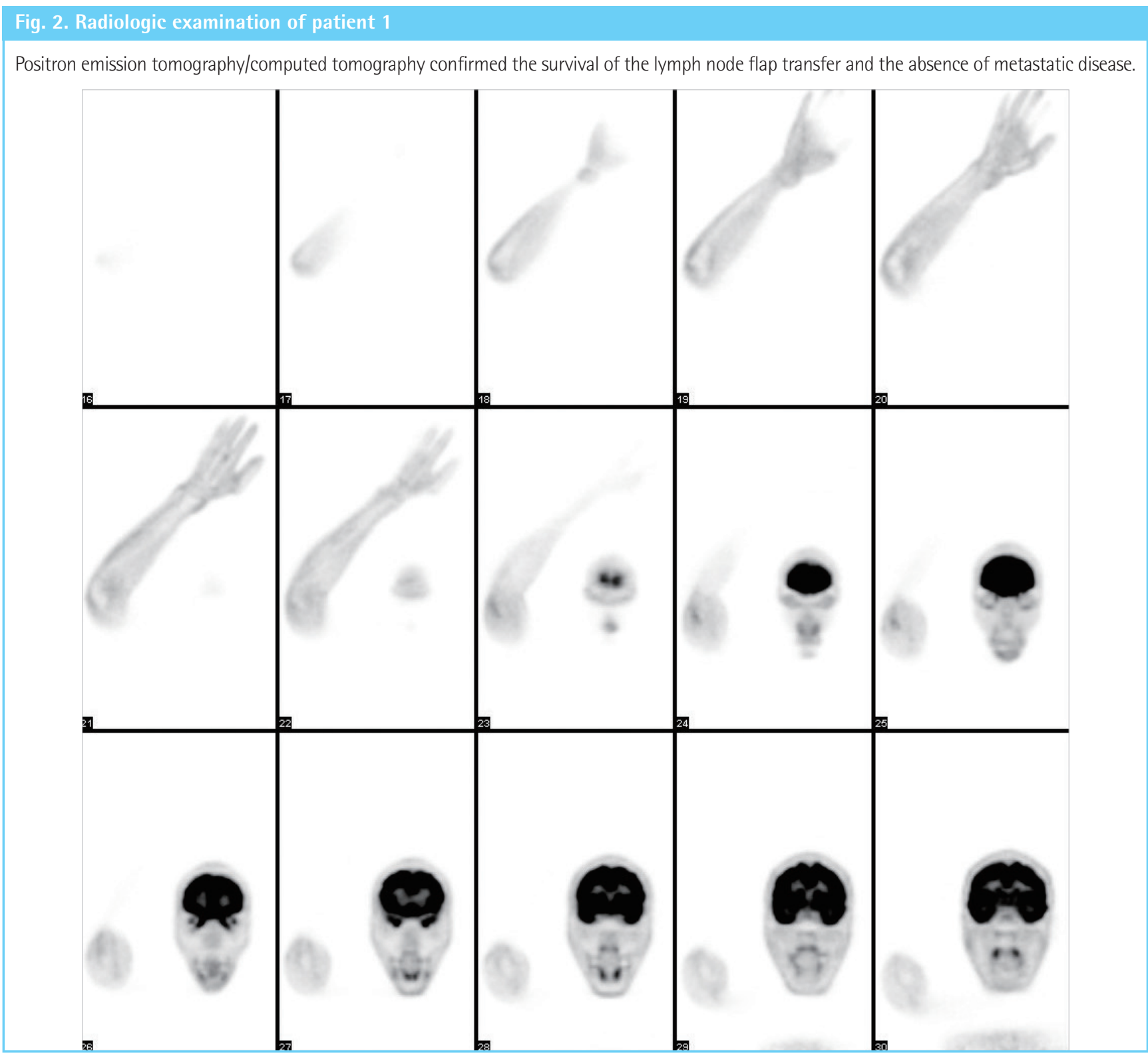

flap (Fig. 2). After nine months of follow-up, no clinical lymphedema, no recurrence, and no metastatic disease were evident (Fig. 3).

\section{Case 2}

A 53-year-old female was admitted with suspected recurrence of melanoma on the right sole of the foot (Fig. 4) and a palpable mass in the inguinal region. A biopsy confirmed the presence of an atypical melanocytic proliferative lesion. Dissection of the ipsilateral groin lymph nodes was performed with a wide local excision (approximately $10 \mathrm{~cm} \times 8 \mathrm{~cm}$ ). The defect was reconstructed with a contralateral groin flap which included the superoinferior lateral lymph nodes (Fig. 5). The superficial circumflex iliac artery was anastomosed to the medial plantar artery

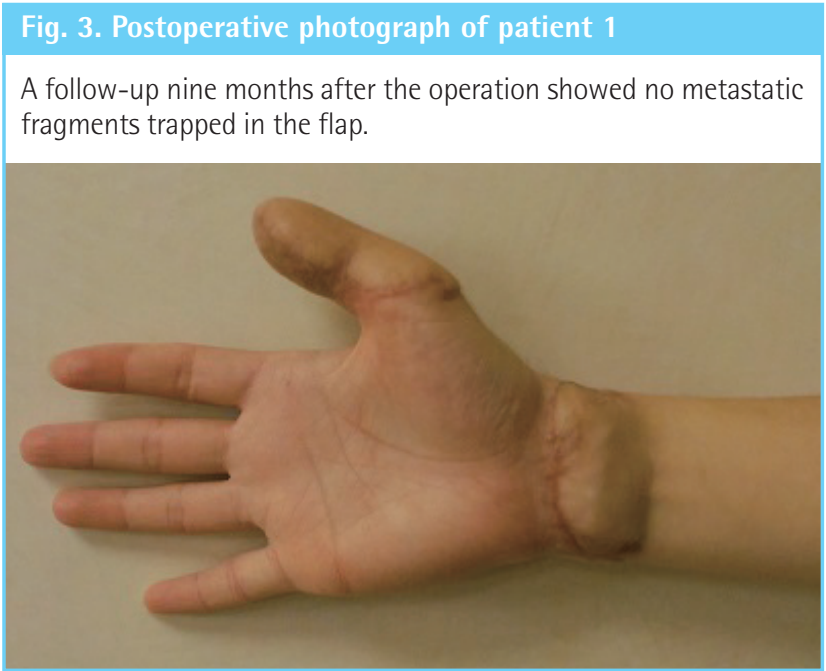




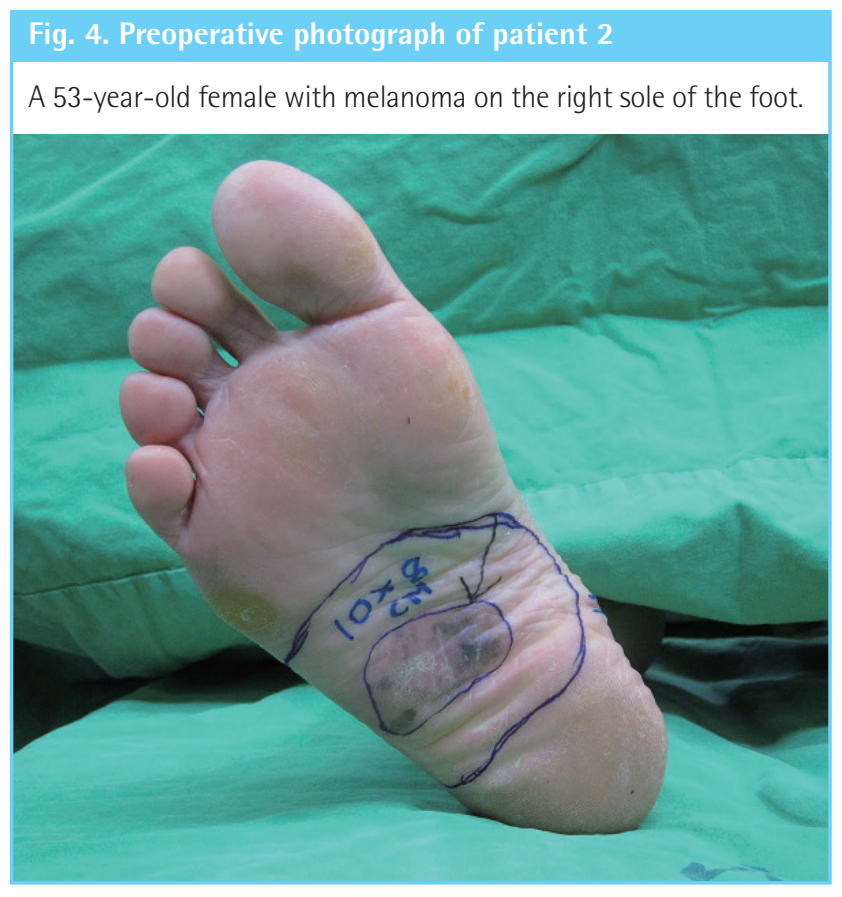

Fig. 5. Intraoperative photograph of patient 2

Reconstruction with a groin flap including the lymph nodes (LN).

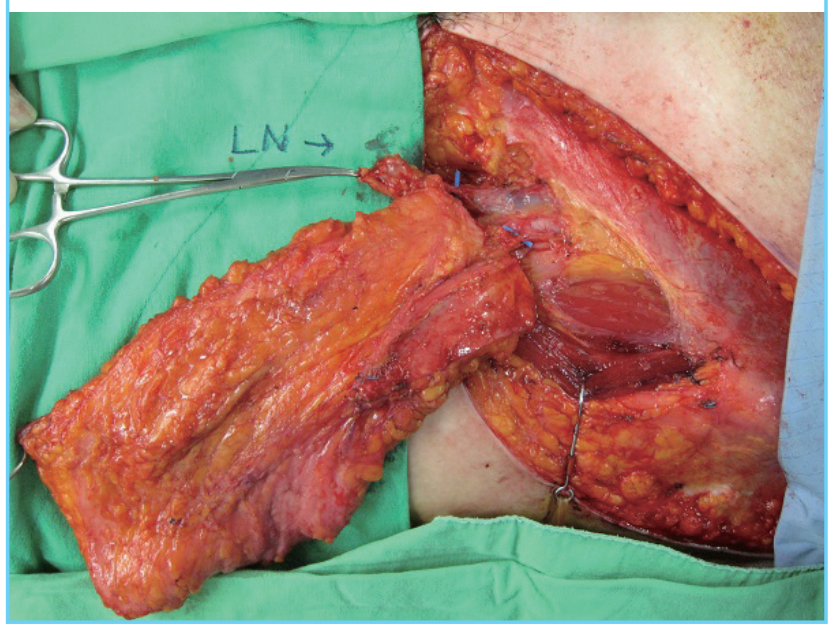

and the superficial circumflex iliac vein was anastomosed to the concomitant vein of the medial plantar artery. An additional vein directing drainage from the lymph nodes was anastomosed to another concomitant vein of the medial plantar artery (Fig. 6). The flap had no complications of note and the patient recovered uneventfully. A postoperative lymphoscyntigraphy exam showed no signs of lymphedema. A PET/CT scan six months after the operation did not detect any metastasis in the flap or in more distal anatomical areas. At a seven-month follow-up examination, the patient was still free of any metastatic disease (Fig. 7).

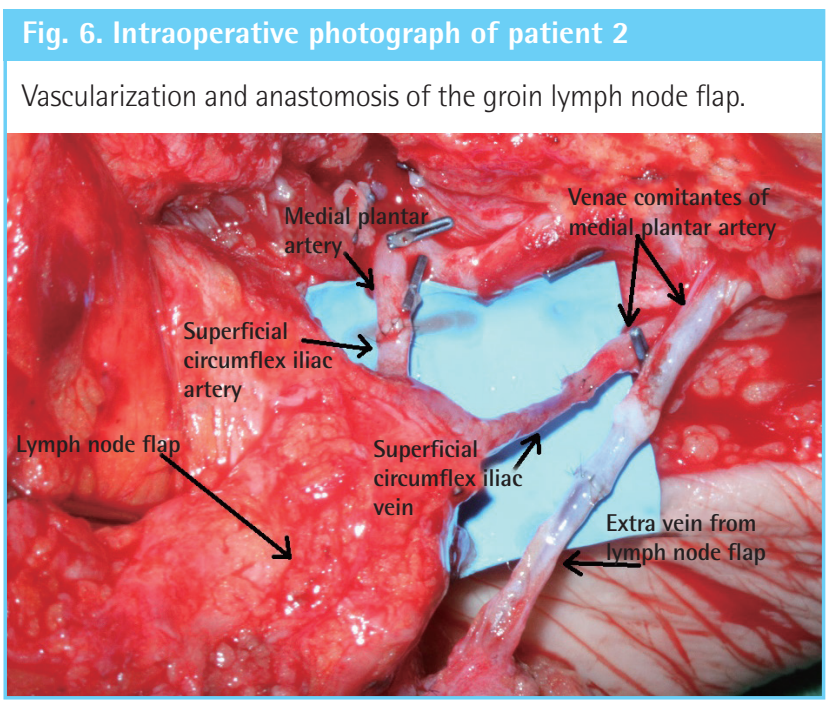

Fig. 7. Postoperative photograph of patient 2

A follow-up examination at seven months showed no recurrence and no metastatic disease.

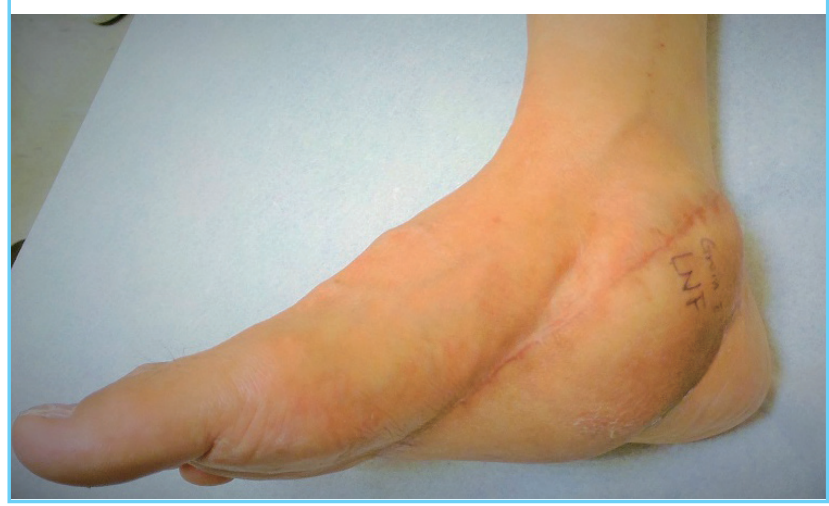

\section{DISCUSSION}

The concept of LNFT was successfully introduced in an animal model by Shesol et al. [10] in 1979 and clinically applied by Clodius et al. [11] in 1982. Since that case report, several studies have confirmed the therapeutic efficacy of this procedure, although multiple explanations have been advanced to explain its mechanism. One theory is that lymphoangiogenesis occurs via growth factors produced by the transplanted lymph nodes and thereby bridges lymphatic pathways $[4,6]$. Another theory is that the transferred lymph nodes act as a lymphatic pump [5].

Additional benefits of lymph node transfer include the stimulation of lymphoangiogenesis and the restoration of lymphatic function, resulting in a lower risk of infection and less pain, as well as preventing lymphedema [1-6]. In this paper, we followed the cases of several patients to examine the potential use of transferred lymph nodes for detecting metastasis after tumor excision. In order to investigate this possibility, we analyzed six patients 


\section{Fig. 8. A postoperative radiologic examination}

(A) Contrast fluid was injected intracutaneously into the distal limb and an infrared camera system was used to obtain images of the superficial lymphatic flow. (B) The superficial lymphatic pattern indicates lymph flow into the transplanted lymph nodes.
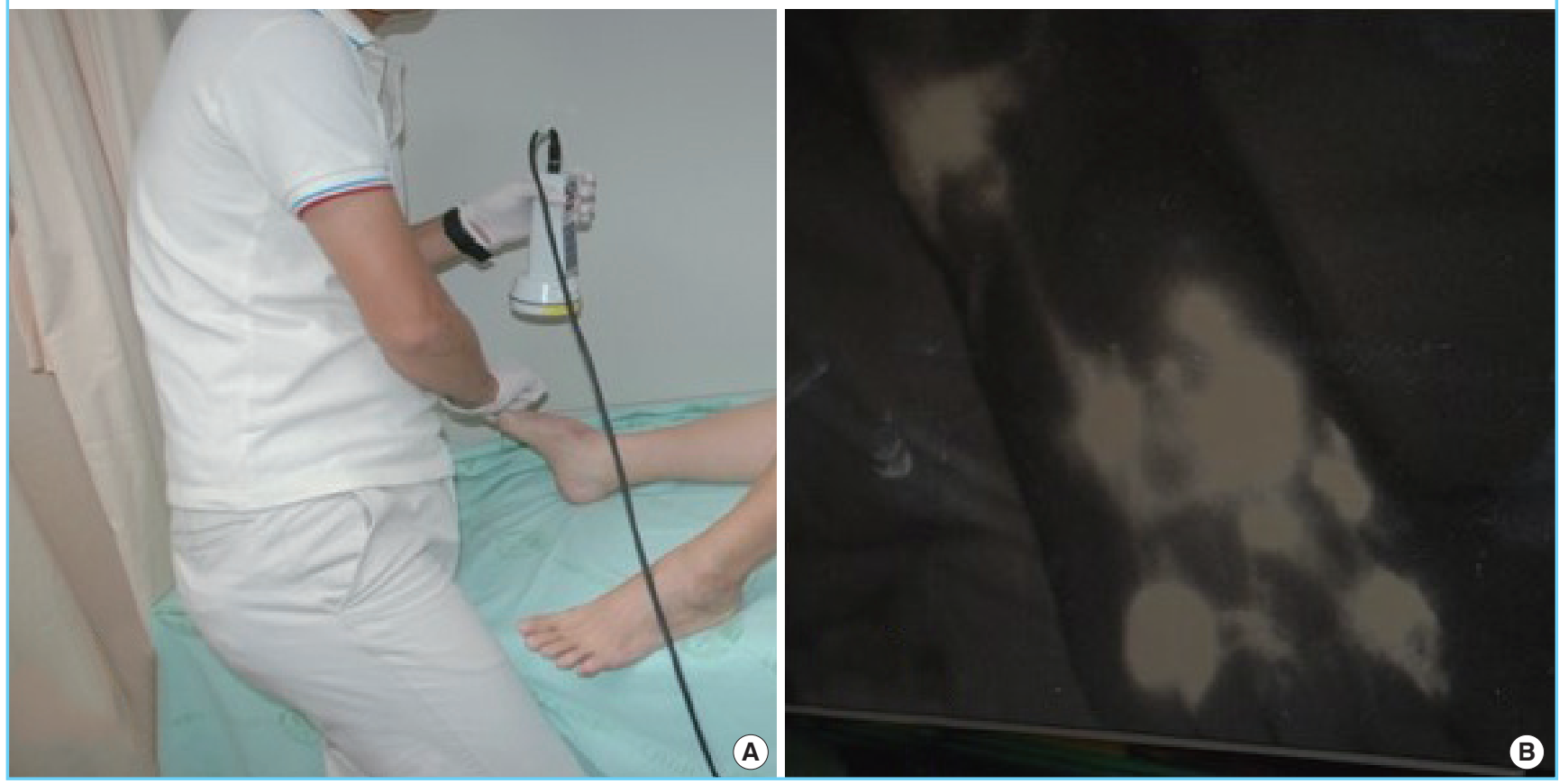

who underwent LNFT. Radiological examinations showed no tumors in the lymph node flaps after six months. Indocyanine green lymphography images showed the pattern of superficial lymphatic flow into the transplanted lymph nodes within a few minutes after injection (Fig. 8).

While our results did not prove that transferred lymph nodes act as metastatic detectors, the possibility certainly warrants further experiment and research. It is important to note that the negative results found in our study may have been influenced by a small patient cohort and a short follow-up time. Further investigation of this topic is particularly intriguing because the management and understanding of the evolution of skin cancers, especially melanoma, remain controversial and treatment protocols are always subject to new revisions [12]. New molecular targets and treatment protocols are constantly under investigation, since the results of most clinical trials are unsatisfactory $[12,13]$.

Metastasis is the most important predictor of a patient's prognosis, and the current focus of treatment is therefore directed toward the unequivocal determination of the presence of metastatic tumors in the adjacent epidermis, sentinel lymph nodes, circulation, and distant sites [13]. The process of metastasis consists of several complex phases and includes multiple mechanisms that have not yet been fully elucidated. Melanoma is theorized to metastasize mainly via the lymphatic route, but the role of lymphatic tissue in the spread and control of the tumor has not yet been defined.

The authors are conscious that this report does not necessarily represent the official view of the National Cancer Institute or the National Institutes of Health, but solely represents a possibility that may eventually be applied in future studies regarding the prognosis of skin cancer. A limitation of this article is the small number of patients analyzed and the short-term follow-up. The use of sentinel lymph nodes for detecting metastasis may be a promising new approach to the management of skin cancer, but we emphasize that further randomized prospective clinical studies, both in vitro and in vivo, are necessary to confirm the possible utility of this surgical procedure. We will continue to monitor our patients and will report whether metastasis is eventually detected via the sentinel lymph nodes in the course of long-term follow-up.

\section{REFERENCES}

1. Becker C, Assouad J, Riquet M, et al. Postmastectomy lymphedema: long-term results following microsurgical lymph node transplantation. Ann Surg 2006;243:313-5.

2. Sapountzis S, Nicoli F, Chilgar R, et al. Evidence-based analysis of lymph node transfer in postmastectomy upper extremity lymphedema. Arch Plast Surg 2013;40:450-1. 
3. Gharb BB, Rampazzo A, Spanio di Spilimbergo S, et al. Vascularized lymph node transfer based on the hilar perforators improves the outcome in upper limb lymphedema. Ann Plast Surg 2011;67:589-93.

4. Saaristo AM, Niemi TS, Viitanen TP, et al. Microvascular breast reconstruction and lymph node transfer for postmastectomy lymphedema patients. Ann Surg 2012;255:468-73.

5. Lin CH, Ali R, Chen SC, et al. Vascularized groin lymph node transfer using the wrist as a recipient site for management of postmastectomy upper extremity lymphedema. Plast Reconstr Surg 2009;123:1265-75.

6. Viitanen TP, Visuri MT, Hartiala P, et al. Lymphatic vessel function and lymphatic growth factor secretion after microvascular lymph node transfer in lymphedema patients. Plast Reconstr Surg Glob Open 2013;1:1-9.

7. Yamamoto T, Yamamoto N, Yamashita M, et al. Efferent lymphatic vessel anastomosis: supermicrosurgical efferent lymphatic vessel-to-venous anastomosis for the prophylactic treatment of subclinical lymphedema. Ann Plast Surg 2014
Nov 11.

8. Soran A, Finegold DN, Brufsky A. Lymphedema prevention and early intervention: a worthy goal. Oncology (Williston Park) 2012;26:249, 54, 56.

9. Aladowicz E, Ferro L, Vitali GC, et al. Molecular networks in melanoma invasion and metastasis. Future Oncol 2013; 9:713-26.

10. Shesol BF, Nakashima R, Alavi A, et al. Successful lymph node transplantation in rats, with restoration of lymphatic function. Plast Reconstr Surg 1979;63:817-23.

11. Clodius L, Smith PJ, Bruna J, et al. The lymphatics of the groin flap. Ann Plast Surg 1982;9:447-58.

12. Morton DL, Thompson JF, Cochran AJ, et al. Final trial report of sentinel-node biopsy versus nodal observation in melanoma. N Engl J Med 2014;370:599-609.

13. Zbytek B, Carlson JA, Granese J, et al. Current concepts of metastasis in melanoma. Expert Rev Dermatol 2008;3:56985 . 